Journal of

Synchrotron

Radiation

ISSN 0909-0495

\section{Macromolecular crystallography radiation damage research: what's new?}

\author{
Elspeth F. Garman ${ }^{\mathrm{a}}$ and Martin Weik ${ }^{\mathrm{b}, \mathrm{c}, \mathrm{d}, \mathrm{e}}$
}

a'Laboratory of Molecular Biophysics, Department of Biochemistry, University of Oxford, South
Parks Road, Oxford OX1 3QU, UK, ' ${ }^{\mathbf{b}}$ Comissariat à I'Energie Atomique, Institut de Biologie
Structurale, F-38054 Grenoble, France, ${ }^{\mathbf{c}}$ CNRS, UMR5075, F-38027 Grenoble, France, ' Université
Joseph Fourier, F-38000 Grenoble, France, and ${ }^{\mathbf{e}}$ ESRF, 6 rue Jules Horowitz, BP 220, 38043
Grenoble Cedex, France. E-mail: elspeth.garman@bioch.ox.ac.uk, martin.weik@ibs.fr

Radiation damage in macromolecular crystallography has become a mainstream concern over the last ten years. The current status of research into this area is briefly assessed, and the ten new papers published in this issue are set into the context of previous work in the field. Some novel and exciting developments emerging over the last two years are also summarized.

During the last ten years the issue of radiation damage in macromolecular crystallography (MX) has become an increasing concern for structural biologists. It is now clear that, even with the crystalline sample held at $100 \mathrm{~K}$ during the data collection, not only do the deleterious effects of damage during diffraction experiments affect the chances of successful structure solution, but they can also compromise the biological information that may be inferred from the results. Researchers interested in understanding the physical and chemical basis of the phenomenon and in investigating ways to reduce the rate of damage have worked on a variety of fronts, and through careful systematic experiments have gained a substantial body of knowledge and a sharp appreciation of the challenges of such studies (see papers from the second, third, fourth and fifth radiation damage workshops in special issues of the Journal of Synchrotron Radiation in 2002, 2005, 2007 and 2009, respectively).

Underpinning this effort has been the development of novel integrated approaches to the research, driven by the need to monitor effects of X-rays on the samples other than solely the global indicators of damage (e.g. overall diffraction strength, fading of highresolution reflections and increasing $B$-factors), and the concomitant degradation of the quality of the electron density obtained, as well as the observed specific structural damage to particularly susceptible amino acids and ligands. This has resulted in new tools, for example online microspectrophotometry, being made available at several synchrotrons. Radiation-induced changes have also been utilized to give new insights into biological pathways and elucidate enzyme mechanisms, for example in carbonic anhydrase (Sjöbloma et al., 2009) and acetylcholinesterase (Colletier et al., 2008).

Articles on radiation damage in MX in this issue build on the accumulating body of research in the field and cover aspects spanning a wide range of approaches. The earliest quantitative experiments on radiation damage in MX were carried out at room temperature (RT) by Blake \& Phillips (1962), who observed an exponential decay of reflection intensities with increasing dose, and devised a model that has endured for 50 years. The paper by Rajendran et al. (2011) reports results using a Pilatus silicon pixel detector and synchrotron beam to measure the RT dose-rate effect previously reported as 'inverse' (i.e. a higher dose tolerance for a higher dose rate) for the range 6-10 $\mathrm{Gy} \mathrm{s}^{-1}$ (Southworth-Davies et al., 2007) for lysozyme crystals. The new results on thaumatin and insulin crystals show that for the range investigated, $\sim 1320 \mathrm{~Gy} \mathrm{~s}^{-1}$ and $\sim 8420 \mathrm{~Gy} \mathrm{~s}^{-1}$, the dose which causes half the total intensity to fade $\left(D_{1 / 2}\right)$ decreased by approximately $75 \%$ over the six-fold increase in dose rate $\left(D_{1 / 2}\right.$ of $0.42 \mathrm{MGy}$ to $0.24 \mathrm{MGy}$ for thaumatin and $0.22 \mathrm{MGy}$ to $0.13 \mathrm{MGy}$ for insulin). Other global indicators [relative Wilson $B$ factors, the redundancy-independent $R$-factor, $I / \sigma(I)$ and the mosaicity] show similar behaviour. As found previously (Blake \& Phillips, 1962), the integrated intensity decreases in an exponential manner. UV-vis spectroscopy is also used by the experimenters to demonstrate that absorptions attributable to disulfide radical anions and 
trapped electrons do not accumulate at high dose rates in the continuous data collection mode of the Pilatus detector.

The above RT study, when taken together with that of Southworth-Davies et al. (2007), implies that there is an optimum dose rate for RT data collection, with the dose tolerance rising with dose rate to a maximum, after which it declines. A third report, which found a $D_{1 / 2}$ for lysozyme crystals at $2800 \mathrm{~Gy} \mathrm{~s}^{-1}$ that was seven times that at $6.0 \mathrm{~Gy} \mathrm{~s}{ }^{-1}$ [0.9 MGy compared with 0.125 MGy (Barker et al., 2009)], is not incompatible with the new results, since the rate of decay has not yet been determined between dose rates of $10 \mathrm{~Gy} \mathrm{~s}^{-1}$ and $2800 \mathrm{~Gy} \mathrm{~s}^{-1}$, and could also pass through a maximum. It should be noted here that the values of $D_{1 / 2}$ reported in the three available studies cover a wide range (lysozyme giving twice that of thaumatin at kGy dose rates), so it seems that dose tolerance of the different proteins is significantly different at room temperature. Thus it would clearly be highly beneficial to determine the value of the dose rate which gives the maximum dose tolerance at room temperature. This is an obvious avenue for further measurements on a larger range of crystals of different proteins, so that some more general guidelines could be established. This is especially pertinent to optimizing results from the recent trend to screen crystals in situ in crystallization trays (Jacquamet et al., 2004).

As well as the RT experiments described above, some interesting recent studies over wider temperature ranges, below $100 \mathrm{~K}$ and up to $300 \mathrm{~K}$, are being conducted. In this issue, Juers \& Weik (2011) report on global and specific radiation damage in crystalline thermolysin at 100 and at $160 \mathrm{~K}$. At $160 \mathrm{~K}$, more amino acid residue types are affected than at $100 \mathrm{~K}$ and $\mathrm{X}$-ray-induced increases in atomic $B$-factors correlate with the proximity of the protein atom to the nearest solvent channel. The results are consistent with increased mobility of the crystal solvent at $160 \mathrm{~K}$; a temperature close to the one $(200 \mathrm{~K})$ above which liquid-like diffusive motions have been described in the solvent of crystalline thaumatin (Warkentin \& Thorne, 2010). Insight into the temperature dependence of radiation damage in the range from $100 \mathrm{~K}$ to room temperature is important for successful slow-cooling of protein crystals (Warkentin \& Thorne, 2009) and for temperature-controlled kinetic crystallography experiments aiming at the generation, trapping and structural characterization of protein intermediate states (Weik \& Colletier, 2010). If radiation damage generally increases upon raising the temperature from $100 \mathrm{~K}$, it is reduced at temperatures below, but details and protection factors are still controversially discussed. Compared with $100 \mathrm{~K}$, specific radiation damage to disulfide bonds has been reported to decrease fourfold at $50 \mathrm{~K}$ in crystalline insulin (Meents et al., 2010) and twofold at $15 \mathrm{~K}$ in crystalline porcine pancreatic elastase (Petrova et al., 2010).

One of the objectives of radiation damage research is to identify practical mitigation strategies, and two papers in this issue report experiments at $100 \mathrm{~K}$ to this end: one investigating the dependence of damage rate on photon energy, and the other testing a radical scavenger. Anecdotal evidence has long been around in MX that the rate of damage is reduced at shorter incident wavelength (higher energy). However, systematic studies of global damage at nine incident energies between 6.4 and $33 \mathrm{keV}$ (Shimizu et al., 2007) and of damage to cadmium metal sites at 6.2 and $12.4 \mathrm{keV}$ (Weiss et al., 2005) have shown no significant differences in either global or metalsite-specific damage at the various incident energies. Some years ago it was pointed out by Arndt (1984) that the ratio of diffracted intensity to energy deposited in the sample does not change appreciably over the range of incident energies used in MX. A new report here (Homer et al., 2011) finds that, although the global damage rate seems to be unchanged, specific damage to lysozyme crystals appears to be greater at $14 \mathrm{keV}$ than at $9 \mathrm{keV}$ for cysteine sulfurs involved in disulfide bridges, although no difference in damage to methionine sulfurs is seen. Although preliminary, this observation points to the potential for minimizing damage to particular specific sites by a judicious choice of incident energy: further investigations will be necessary to endorse these results.

In attempts to reduce damage rates, effective radical scavengers for MX at $100 \mathrm{~K}$ have been sought by various researchers with mixed and sometimes controversial results (Murray \& Garman, 2002; Kauffmann et al., 2006; Nowak et al., 2009; Macedo et al., 2009; Southworth-Davies \& Garman, 2007) and also at RT (Barker et al., 2009). In this issue a highly efficient electron scavenger, sodium nitrate, is found to reduce specific structural damage to disulfide bonds by a factor of five at $100 \mathrm{~K}$ and to decrease the rate of global damage (as measured from the total integrated intensity of sequential datasets on lysozyme crystals) by a factor of two (de la Mora et al., 2011). For the first time the radiation chemistry of a scavenger protection mechanism can be seen in the electron density maps, as the nitrate ion is seen to cleave an oxygen atom to leave $\mathrm{NO}_{2}$, and only when the $\mathrm{NO}_{2}$ disappears from the electron density is the disulfide bond damaged. Other results in this paper strengthen the growing evidence that ascorbate is an effective scavenger: ascorbate is observed bound in the crystal and oxidative damage is inhibited (i.e. glutamates and aspartates were protected from decarboxylation), though, as expected, no signs of consequent reduction of ascorbate is observed, as can be rationalized from the chemistry of the system. Furthering our understanding of the mechanism of action of the various scavengers already identified for MX should allow a more rational approach to their general use.

In the scavenger study described above, online microspectrophotometry is used to observe that the addition of nitrate quenched the disulfide anion radical peak observable at $400 \mathrm{~nm}$ upon irradiation and also the solvated electron peak at around $580 \mathrm{~nm}$. Such spectroscopic measurements can provide vital complementary information on crystalline biological macromolecules and the associated toolbox now includes UV-vis absorption, fluorescence, resonant and nonresonant Raman, IR, EPR and XAS spectroscopic techniques. Online UV-vis absorption, fluorescence and Raman spectroscopic techniques are available occasionally to MX users at the ESRF (McGeehan et al., 2009, 2011) and the SLS (Owen, Pearson et al., 2009), and Orville et al. (2011) now report on the routine availability of these three techniques at the NSLS 
beamline X26-C and on their control being intimately linked into the beamline operation. These authors also prepare the ground for routinely integrating spectroscopic characteristics into a Protein Data Bank deposition. In another paper in this issue, online UV-vis microspectrophotometry is employed to examine X-ray-induced reduction in metalloproteins (Owen et al., 2011). In particular, these experimenters show that reduction of the haem centre in crystalline myoglobin and cytochrome $c$ is independent of the rate at which the X-ray dose is absorbed by the sample at $100 \mathrm{~K}$. Online UV-vis microspectrophotometry has also been essential to the success of a crystallographic study in which a nitrite adduct of ferric myoglobin has been generated, trapped and characterized (Yi et al., 2010). A combination of Raman spectroscopy and MX has recently provided evidence for X-ray-induced repair mechanisms of disulfide radical anions in crystalline lysozyme (Carpentier et al., 2010) and of an X-ray-induced intermediate state in the fluorescent protein IrisFP (Adam et al., 2009). Furthermore, IR spectroscopy on butyrylcholinesterase crystals suggested the generation of $\mathrm{CO}_{2}$ upon X-ray irradiation (Sage et al., 2011), thus complementing the experimental evidence for hydrogen formation during X-ray irradiation (Meents et al., 2010). The diversifying toolbox of spectroscopic methods available to the crystallographer, and their widespread implementation at synchrotron sources worldwide, will continue to sharpen and facilitate our ability to characterize macromolecular crystal structures.

As mentioned above, radiation damage can compromise successful structure solution. However, as shown by Ravelli et al. (2003), it can also be utilized to obtain phases (RIP; radiation-damage-induced phasing) by collecting data before and after an X-ray 'burn' which damages the disulfide bridges and other specific sites. Alternatively, UV radiation can be used to induce the necessary damage instead of X-rays (Nanao \& Ravelli, 2006). Here de Sanctis et al. (2011) demonstrate that the isomorphous signal obtained from selenium sites in a protein crystal before and after irradiation with UV light (using a $266 \mathrm{~nm}$ laser) can provide enough signal, when combined with the anomalous signal measured at the selenium absorption edge, to give phases for structure solution by SIRAS (single isomorphous replacement anomalous scattering). They compare the results from this procedure with those from a more conventional MAD experiment on the same two proteins, and conclude that their method could be useful for problematic cases of selenium phasing. The description of the measurements complements a recent report by the same group (Panjikar et al., 2011), in which they describe the solution of three different selenium derivatized protein structures by RIP alone with UV-irradiation using data collected at an energy $(12.0 \mathrm{keV})$ far from the absorption edge of selenium. A tunable beamline is thus unnecessary for the experiments, and the method could straightforwardly be used on a home source.

For the radiation damage research effort to bear fruit, the knowledge gained from it must be used to enable the investigators to routinely optimize the diffraction experiment and obtain the required data from their crystal before it is too damaged. Thus easy-to-use online tools, both predictive and diagnostic, must be developed. Steps in this direction are reported here in a contribution by Leal et al. (2011), which complements their previous work on the software program BEST (Bourenkov \& Popov, 2010). BEST models the particular diffraction experiment and can optimize the data collection strategy, taking radiation damage into account by making the assumption that it proceeds at a rate of $B$-factor change of $1 \AA^{2} \mathrm{MGy}^{-1}$ for $100 \mathrm{~K}$ data collection: an average value arrived at following extensive experimental validation (Kmetko et al., 2006). In the new development an automatic procedure for characterizing the radiation sensitivity of crystals has been incorporated into the EDNA online data analysis framework (Incardona et al., 2009) and the ESRF online data collection software $M x C u B E$. The procedure, which involves several cycles of a thin wedge of data collection and then a burn to induce significant $B$-factor changes in a sacrificial crystal, enables the experimenter to determine the dose that reduces the outer-resolution shell by a factor of three (around $10 \mathrm{MGy}$ for a strongly diffracting crystal). The results of this experiment can then be supplied to BEST so that the data collection strategy can be optimized. It is important to note that benefit can be gained not only by optimizing the data collection protocol but also by taking care to properly treat data showing damage characteristics at the data reduction stage (Borek et al., 2010).

For experimenters at the beamline there is a lack of clear guidance and simple radiation damage minimization procedures for use in practice. How well do those that are available perform? To answer this question a statistically significant number of datasets from 'real life' datasets collected by a structural genomics group focusing on solving the structures of human proteins has been analysed in detail to characterize their manifestations of radiation damage (Krojer \& von Delft, 2011). The results are highly illuminating and present a clear challenge to the radiation damage research community to try to better characterize the diffraction experiment, so that the predictive power of the current software tools can be improved, and to beamline scientists to provide more accurate information to experimenters on the flux density distribution of the beam.

As is clear from the above study, a vital component that underpins the advance in radiation damage studies is the necessary development of better tools to enable damage indicators to be plotted against a metric that allows easy comparisons of results from different experiments. The most convenient metric for this purpose is the absorbed dose, but accurate quantitation of dose depends critically on a reliable knowledge of the beam parameters (energy, size, profile and flux) and the crystal characteristics (size, and constituent atom types and their number). Regular calibration of flux is now routine at some beamlines and advances have been made in simplifying flux calibration methodology (Owen, Holton et al., 2009), although the beam shape is often unknown [see the results presented here by Krojer \& von Delft (2011)]. There have also been recent improvements to RADDOSE, a program that allows experimenters to conveniently calculate 
the dose from the experimental parameters (Paithankar et al., 2009; Paithankar \& Garman, 2010; Murray et al., 2004). However, RADDOSE still gives doses that are too low if the crystal is bigger than the beam size, as it does not yet take into account the rotation of the crystal, which brings unexposed material into the beam during the experiment. This means that calculation of dose for experiments using microbeams is particularly poor. For a proper solution to this problem, which is currently being addressed, the crystal shape and its orientation with respect to the beam and rotation axes will have to be parameterized and passed online to the program. This presents a number of challenges to be overcome in the future.

A dialogue between scientists in the fields of MX and of electron diffraction and imaging concerning radiation damage issues was only initiated recently (Massover, 2007). An obstacle to this dialogue has been the use of different measures to quantify the dose absorbed by specimens in the two fields. In MX, the absorbed dose is given in gray ( $\mathrm{Gy}=$ $\mathrm{J} \mathrm{kg}^{-1}$ ) as the absorbed energy per unit mass, whereas the integrated incident flux per unit area (in $\mathrm{e}^{-} \AA^{-2}$ ) is used in electron diffraction and imaging. The paper by Karuppasamy et al. (2011) now bridges the gap by converting integrated incident electron fluxes into gray, thus paving the way for a systematic comparison between radiation damage issues in MX and in electron diffraction and imaging. Karuppasamy et al. (2011) determine that the typical dose required to collect a single micrograph in single-particle cryo-electron microscopy (with an integrated incident flux per unit area of $25 \mathrm{e}^{-} \AA^{-2}$ per image) corresponds to the experimental dose limit which should ideally not be exceeded per data set in MX [i.e. $30 \mathrm{MGy}$ (Owen et al., 2006)]. In contrast to cryo-MX, Karuppasamy et al. (2011) show that radiation damage in single-particle cryo-electron microscopy depends significantly on the dose rate, i.e. on the time interval during which a given dose is absorbed by the specimen. As in MX, the temperature dependence of radiation damage in electron imaging has also been investigated (Bammes et al., 2010), in the range from 4 to $100 \mathrm{~K}$. The authors conclude that $100 \mathrm{~K}$ is the optimum data collection temperature in single-particle cryo-electron microscopy at high resolution (4-20 $)$ ), whereas intermediate temperatures ( 25 and $42 \mathrm{~K}$ ) are optimal in lower-resolution (20-60 A) studies, such as in cryo-electron tomography. A further exchange on radiation damage mechanisms and effects between macromolecular crystallographers and electron microscopists will mutually benefit both fields.

There have been some additional significant reports on radiation damage in MX and related matters since the last Journal of Synchrotron Radiation special issue on radiation damage two years ago, including interesting material presented at the 2009 CCP4 Study Weekend on 'Experimental Phasing and Radiation Damage' that was published in the May 2010 edition of Acta Crystallographica Section D. Among the new technical developments in MX is the availability of microbeams at increasing numbers of synchrotron sites. Some years ago, Nave \& Hill (2005) and Cowan \& Nave (2008) showed through Monte Carlo simulation studies that radiation damage might be reduced if a microbeam $(<10 \mu \mathrm{m})$ in conjunction with higher-energy incident X-rays was used, since a non-negligible proportion of the photoelectrons would emerge from edges of the irradiated volume and no longer contribute to the ('umbral') absorbed dose. This idea has now been experimentally tested with a $1 \mu \mathrm{m} 18.5 \mathrm{keV}$ beam at the APS, and the results have been reported very recently in a paper by Sanishvili et al. (2011), who show that the damage rate normalized for dose was reduced by a factor of three from the largest $(15.6 \mu \mathrm{m})$ to the smallest $(0.84 \mu \mathrm{m}) \mathrm{X}$-ray beam tested. The damage extended up to $4 \mu \mathrm{m}$ from the centre of the beam, giving information on the range of the photoelectrons and on the extent of the unexposed volume affected by the 'penumbral' dose. These results give clear indications of ways to optimize the use of microbeams in MX.

Two recent landmark papers might open a new era for structural biology (Chapman et al., 2011; Seibert et al., 2011), in which highly brilliant beams from X-ray free-electron lasers (XFELs) are employed to study nanocrystals, or even single protein molecules or fragile complexes thereof. The underlying principle is 'diffraction before destruction', in which diffraction using ultrashort X-ray pulses of several tens of femtoseconds in length occurs before the sample disintegrates by Coulomb explosion (Neutze et al., 2000). The study by Chapman et al. (2011) on nanocrystals of photosystem I provides the first experimental evidence that femtosecond diffraction can indeed outrun radiation damage. A 70 ps pulse of $1.8 \mathrm{keV}$ X-rays delivered by the LCLS XFEL in Stanford did not lead to noticeable radiation damage in the diffraction patters at $8.5 \AA$ resolution. The absorbed dose was 700 MGy per pulse, more than 20 times the experimental limit of 30 MGy mentioned above. With two more XFELs coming online in the next few years (the European XFEL in Hamburg, Germany, and the SPring-8 Compact SASE Source in Japan), there is great hope for exploring radiation damage in hitherto inaccessible dose ranges, time scales and sizes.

We thank Ian Carmichael and Arwen Pearson for providing useful comments on this manuscript. We are also very grateful for the local organisational skills of Ana Gonzales and the generosity of the Stanford Synchrotron Radiation Laboratory in hosting the Sixth International Workshop on X-ray Damage to Crystalline Biological Samples, during which most of the work in these special issue papers was presented. Funding for this Workshop was made possible (in part) by 1R13RR03072601 from the National Center for Research Resources, and from the National Institute of General Medical Sciences. The views expressed in written conference materials or publications and by speakers and moderators do not necessarily reflect the official policies of the Department of Health and Human Services; nor does mention by trade names, commercial practices or organizations imply endorsement by the US Government. Funding was also provided by the US Department of Energy, Office of Biological and Environmental Research; Bio-X, Stanford University; Genentech, Inc.; and the Commissariat à l'Energie Atomique, France. 


\section{References}

Adam, V., Carpentier, P., Violot, S., Lelimousin, M., Darnault, C., Nienhaus, G. U. \& Bourgeois, D. (2009). J. Am. Chem. Soc. 131, 18063-18065.

Arndt, U. W. (1984). J. Appl. Cryst. 17, 118-119.

Bammes, B. E., Jakana, J., Schmid, M. F. \& Chiu, W. (2010). J. Struct. Biol. 169, 331-341.

Barker, A. I., Southworth-Davies, R. J., Paithankar, K. S., Carmichael, I. \& Garman, E. F. (2009). J. Synchrotron Rad. 16, 205-216.

Blake, C. C. F. \& Phillips, D. C. (1962). Biological Effects of Ionizing Radiation at the Molecular Level, pp. 183-191. Vienna: International Atomic Energy Agency.

Borek, D., Cymborowski, M., Machius, M., Minor, W. \& Otwinowski, Z. (2010). Acta Cryst. D66, 426-436.

Bourenkov, G. P. \& Popov, A. N. (2010). Acta Cryst. D66, 409-419.

Carpentier, P., Royant, A., Weik, M. \& Bourgeois, D. (2010). Structure, 18, 1410-1419.

Chapman, H. N. et al. (2011). Nature (London), 470, 73-77.

Colletier, J. P., Bourgeois, D., Sanson, B., Fournier, D., Sussman, J. L., Silman, I. \& Weik, M. (2008). Proc. Natl Acad. Sci. USA, 105, 11742-11747.

Cowan, J. A. \& Nave, C. (2008). J. Synchrotron Rad. 15, 458-462.

De la Mora, E., Carmichael, I. \& Garman, E. F. (2011). J. Synchrotron Rad. 18, 346-357.

Homer, C., Cooper, L. \& Gonzalez, A. (2011). J. Synchrotron Rad. 18, 338-345.

Incardona, M.-F., Bourenkov, G. P., Levik, K., Pieritz, R. A., Popov, A. N. \& Svensson, O. (2009). J. Synchrotron Rad. 16, 872-879.

Jacquamet, L., Ohana, J., Joly, J., Borel, F., Pirocchi, M., Charrault, P., Bertoni, A., Israel-Gouy, P., Carpentier, P., Kozielski, F., Blot, D. \& Ferrer, J. L. (2004). Structure, 12, 1219-1225.

Juers, D. H. \& Weik, M. (2011). J. Synchrotron Rad. 18, 329-337.

Karuppasamy, M., Nejadasl, F. K., Vulovic, M., Koster, A. J. \& Ravelli, R. B. G. (2011). J. Synchrotron Rad. 18, 398-412.

Kauffmann, B., Weiss, M. S., Lamzin, V. S. \& Schmidt, A. (2006). Structure, 14, 1099-1105.

Kmetko, J., Husseini, N. S., Naides, M., Kalinin, Y. \& Thorne, R. E. (2006). Acta Cryst. D62, 1030-1038.

Krojer, T. \& von Delft, F. (2011). J. Synchrotron Rad. 18, 387-397.

Leal, R. M. F., Bourenkov, G. P., Svensson, O., Spruce, D., Guijarro, M. \& Popov, A. N. (2011). J. Synchrotron Rad. 18, 381-386.

Macedo, S., Pechlaner, M., Schmid, W., Weik, M., Sato, K., Dennison, C. \& Djinović-Carugo, K. (2009). J. Synchrotron Rad. 16, 191204.

McGeehan, J. E., Bourgeois, D., Royant, A. \& Carpentier, P. (2011). Biochim. Biophys. Acta. In the press.

McGeehan, J., Ravelli, R. B. G., Murray, J. W., Owen, R. L., Cipriani, F., McSweeney, S., Weik, M. \& Garman, E. F. (2009). J. Synchrotron Rad. 16, 163-172.

Massover, W. H. (2007). J. Synchrotron Rad. 14, 116-127.

Meents, A., Gutmann, S., Wagner, A. \& Schulze-Briese, C. (2010). Proc. Natl Acad. Sci. USA, 107, 1094-1099.

Murray, J. \& Garman, E. (2002). J. Synchrotron Rad. 9, 347-354.
Murray, J. W., Garman, E. F. \& Ravelli, R. B. G. (2004). J. Appl. Cryst. 37, 513-522.

Nanao, M. H. \& Ravelli, R. B. (2006). Structure, 14, 791-800.

Nave, C. \& Hill, M. A. (2005). J. Synchrotron Rad. 12, 299-303.

Neutze, R., Wouts, R., van der Spoel, D., Weckert, E. \& Hajdu, J. (2000). Nature (London), 406, 752-757.

Nowak, E., Brzuszkiewicz, A., Dauter, M., Dauter, Z. \& Rosenbaum, G. (2009). Acta Cryst. D65, 1004-1006.

Orville, A. M., Buono, R., Cowan, M., Héroux, A., Shea-McCarthy, G., Schneider, D. K., Skinner, J. M., Skinner, M. J., Stoner-Ma, D. \& Sweet, R. M. (2011). J. Synchrotron Rad. 18, 358-366.

Owen, R. L., Holton, J. M., Schulze-Briese, C. \& Garman, E. F. (2009). J. Synchrotron Rad. 16, 143-151.

Owen, R. L., Pearson, A. R., Meents, A., Boehler, P., Thominet, V. \& Schulze-Briese, C. (2009). J. Synchrotron Rad. 16, 173-182.

Owen, R. L., Rudino-Pinera, E. \& Garman, E. F. (2006). Proc. Natl Acad. Sci. USA, 103, 4912-4917.

Owen, R. L., Yorke, B. A., Gowdy, J. A. \& Pearson, A. R. (2011). J. Synchrotron Rad. 18, 367-373.

Paithankar, K. S. \& Garman, E. F. (2010). Acta Cryst. D66, 381-388.

Paithankar, K. S., Owen, R. L. \& Garman, E. F. (2009). J. Synchrotron Rad. 16, 152-162.

Panjikar, S., Mayerhofer, H., Tucker, P. A., Mueller-Dieckmann, J. \& de Sanctis, D. (2011). Acta Cryst. D67, 32-44.

Petrova, T., Ginell, S., Mitschler, A., Kim, Y., Lunin, V. Y., Joachimiak, G., Cousido-Siah, A., Hazemann, I., Podjarny, A., Lazarski, K. \& Joachimiak, A. (2010). Acta Cryst. D66, 1075-1091.

Rajendran, C., Dworkowski, F. S. N., Wang, M. \& Schulze-Briese, C. (2011). J. Synchrotron Rad. 18, 318-328.

Ravelli, R. B., Leiros, H. K., Pan, B., Caffrey, M. \& McSweeney, S. (2003). Structure, 11, 217-224.

Sage, J. T., Zhang, Y., McGeehan, J., Ravelli, R., Weik, M. \& van Thor, J. J. (2011). Biochim. Biophys. Acta. In the press.

Sanctis, D. de, Tucker, P. A. \& Panjikar, S. (2011). J. Synchrotron Rad. 18, 374-380.

Sanishvili, R., Yoder, D. W., Pothineni, S. B., Rosenbaum, G., Xu, S., Vogt, S., Stepanov, S., Makarov, O. A., Corcoran, S., Benn, R., Nagarajan, V., Smith, J. L. \& Fischetti, R. F. (2011). Proc. Natl Acad. Sci. USA. In the press.

Seibert, M. M. et al. (2011). Nature (London), 470, 78-81.

Shimizu, N., Hirata, K., Hasegawa, K., Ueno, G. \& Yamamoto, M. (2007). J. Synchrotron Rad. 14, 4-10.

Sjöbloma, B., Polentarutti, M. \& Djinović-Carugo, K. (2009). Proc. Natl Acad. Sci. USA, 106, 10609-10613.

Southworth-Davies, R. J. \& Garman, E. F. (2007). J. Synchrotron Rad. 14, 73-83.

Southworth-Davies, R. J., Medina, M. A., Carmichael, I. \& Garman, E. F. (2007). Structure, 15, 1531-1541.

Warkentin, M. \& Thorne, R. E. (2009). J. Appl. Cryst. 42, 944-952.

Warkentin, M. \& Thorne, R. E. (2010). Acta Cryst. D66, 1092-1100.

Weik, M. \& Colletier, J.-P. (2010). Acta Cryst. D66, 437-446.

Weiss, M. S., Panjikar, S., Mueller-Dieckmann, C. \& Tucker, P. A. (2005). J. Synchrotron Rad. 12, 304-309.

Yi, J., Orville, A. M., Skinner, J. M., Skinner, M. J. \& Richter-Addo, G. B. (2010). Biochemistry, 49, 5969-5971. 